\title{
Statistical Modelling
}

http://smj.sagepub.com/

\section{A short note on quantifying and visualizing yearly variation in online monitored temperature data \\ Göran Kauermann and Thomas Mestekemper \\ Statistical Modelling 2012 12: 195 \\ DOI: $10.1177 / 1471082 \times 1001200204$}

The online version of this article can be found at:

http://smj.sagepub.com/content/12/2/195

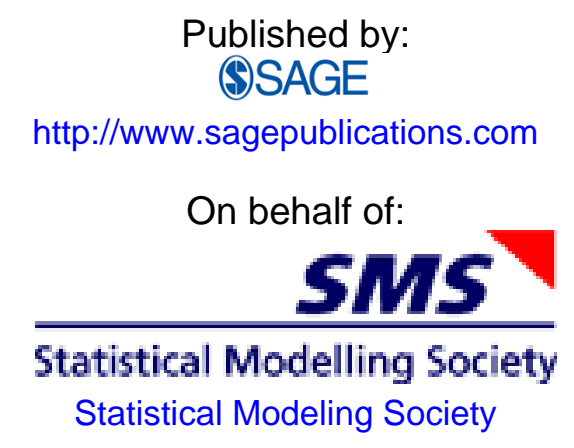

Additional services and information for Statistical Modelling can be found at:

Email Alerts: http://smj.sagepub.com/cgi/alerts

Subscriptions: http://smj.sagepub.com/subscriptions

Reprints: http://www.sagepub.com/journalsReprints.nav

Permissions: http://www.sagepub.com/journalsPermissions.nav

Citations: http://smj.sagepub.com/content/12/2/195.refs.html

>> Version of Record - Apr 17, 2012

What is This? 


\title{
A short note on quantifying and visualizing yearly variation in online monitored temperature data
}

\author{
Göran Kauermann ${ }^{1}$ and Thomas Mestekemper ${ }^{2}$ \\ ${ }^{1}$ Department of Statistics, Ludwig-Maximilians-University Munich, Germany \\ ${ }^{2}$ University Bielefeld, Center for Statistics, Bielefeld, Germany
}

\begin{abstract}
The paper demonstrates how seasonal variation in sequentially arriving temperature data can be visualized by the specification of landmarks and subsequent time warping. We exemplify the idea with water temperature data from the river Wupper in northwestern Germany and with air temperature data from Berlin, Germany. Landmarks are thereby based on temperature thresholds. The method allows to assess whether the seasonal variation is running ahead or behind the average.
\end{abstract}

Key words: warping; landmark specification; monotonic smoothing; temperature curves

Received June 2010; revised November 2010; accepted February 2011

\section{Introduction}

We consider yearly water and air temperature variation, respectively. For the first, we analyze data from the river Wupper in the northwestern part of Germany. For the second, we look at air temperature taken in Berlin, Germany. For both examples we focus the question how to identify variation of the annual temperature course. Evidently, not all years are equal and even though the seasons are the dominating factor, the beginning and end of warm and cold temperature seasons are variable and can shift between years. In this paper, we focus on the problem of assessing from a statistical perspective whether in a particular year the season is running behind or ahead of the 'average year'. Note that the 'average temperature' in an average year has to be defined on both axes. First, the temperature curve showing the average temperature at each time point. Second, the time axis which shows a standardized time scale which for a particular year is stretched or compressed over some periods of the year. In this paper, we are primarily interested in the second component, i.e., derivations form the 'average year' in terms of stretching or compressing time.

Address for correspondence: Göran Kauermann, Department of Statistics, Ludwig-MaximiliansUniversity Munich, Ludwigstrasse 33, 80539 Munich, Germany.

E-mail: Georan.kauermann@stat.uni-muenchen.de; gkauermann@wiwi-uni-bielefeld.de

(C) 2012 SAGE Publications

$10.1177 / 1471082 X 1001200204$ 


\section{Göran Kauermann and Thomas Mestekemper}

The data of the first example give upstream water temperature which are not affected by any kind of (relevant) human interference. The curves of the maximum daily water temperatures are shown in Figure 1 with observations being shifted vertically (by $10^{\circ} \mathrm{C}$ ) for better visual impression. Years are fixed here to last from 1 July to 30 June instead of the Julian calendar, which is more coherent with the seasonal and biological pattern. Just by pure visual inspection we see a number of features. For instance, summer 2003 started early (mid May) and lasted long (until September), or winter 2005 started early (mid November). Our intention is to get statements like these more formally based on statistical grounds.

The data we consider are functional. Functional data analysis is a term coined by Ramsay and Silverman (2005) and recently further discussed in Ferraty and Vieu (2006) and Ramsay et al. (2009). The problem we are tackling is also known under terms like warping or registration. An early reference is Kneip and Gasser (1992) or Gasser and Kneip (1995). They define landmarks by assuming that the data are individual smooth functions disturbed by noise. The individual functions are then used to reveal common structures like local maxima and minima which are then defined as landmarks. Silverman (1995) discusses additive shifts on the time scale, see also Rønn (2001). Ramsay and Li (1998) and Gervini and Gasser (2004) suggest an automatic warping or also called self-modelling warping. They look at vertical and horizontal deviations simultaneously. A wavelet approach in this direction has been proposed in Bigot (2006). More recently Liu and Yang (2009) describe a procedure for simultaneous registration and clustering of curves.

The data structure and the focus of our data analysis do not allow for a direct application of the methods described above because of the following reasons. First, looking at our data it appears that the only clearly exposed local extrema mark summer and winter and other local extrema appear more as spurious events. Hence, the underlying ideas of finding local extrema as suggested in Gasser and Kneip (1995) seem not fruitful except of defining these two yearly extrema. Second, all methods described above are designed for a retrospective point of view, that is, the complete data of an observation period are necessary to run a warping or registration procedure. In our example, this would mean, based on the data of a year we could retrospectively decide whether seasons were running ahead or behind the 'average year'. We want, however, a procedure reacting 'online' by looking at recent measurements. Hence, based on data collected in the progression over a year, we want to decide whether the temperature is running ahead or behind the average. Third, our yearly observations are not independent replicates but consecutive, that is the end of one year is the beginning of the next year. In so far, yearly warping functions must connect continuously. To overcome these issues we suggest to find landmarks in a data-driven style and to retrieve structures which can be found quite reliably every year. To do so, we consider the daily average temperatures (see, e.g., Figure 1) and employ statistical tests to recent temperature measurements in order to decide whether a pre-specified temperature threshold has been significantly exceeded or fallen below. By only looking at small time windows of recently available temperature values we can justify the 'online' applicability of our method. 


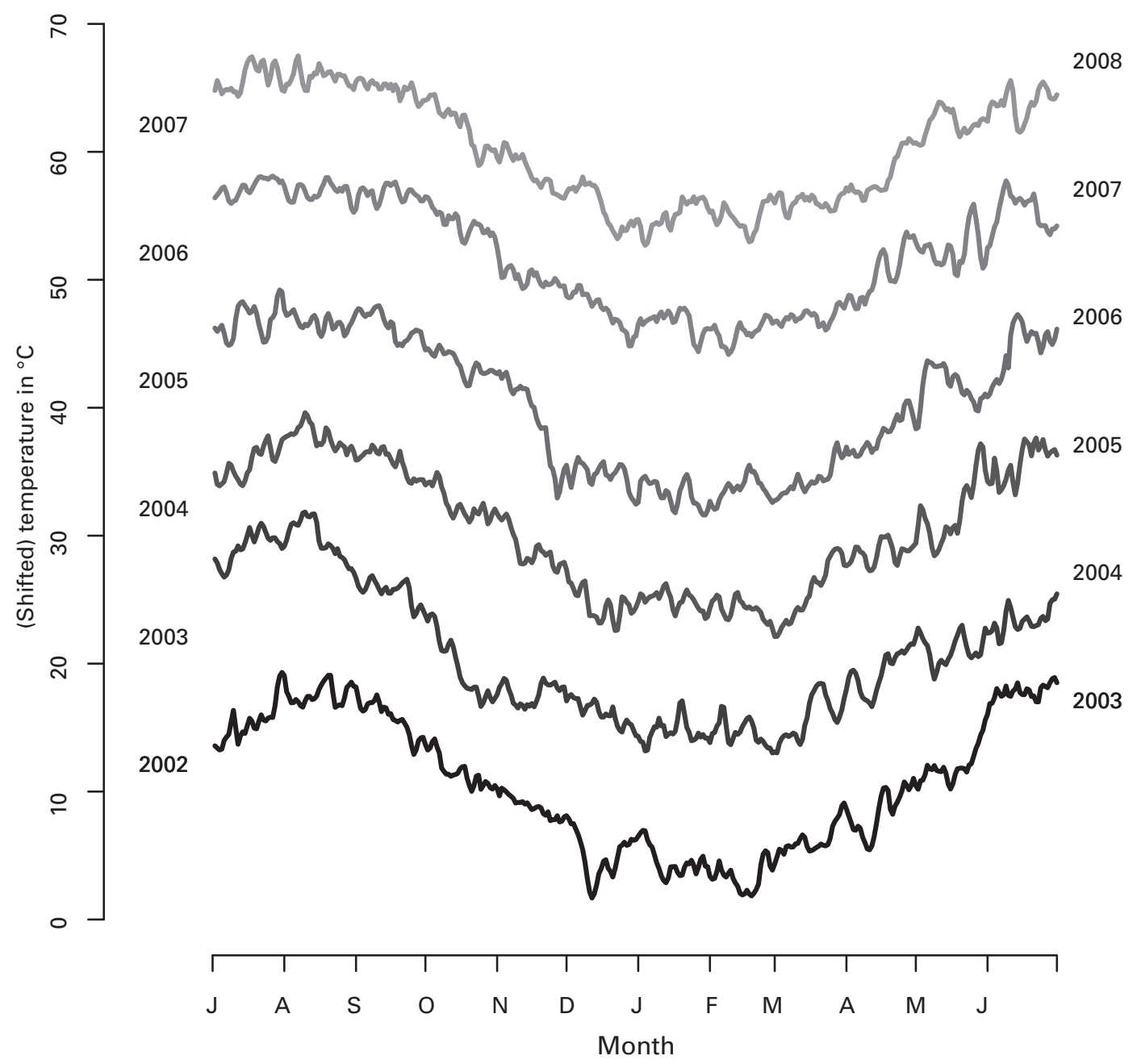

Figure 1 Daily average water temperature recorded from 1 July 2002 to 30 June 2008. For visualization purposes, the curves from the second year onwards have been shifted by cumulatively adding $10^{\circ} \mathrm{C}$

The paper is organized as follows. In Section 2, we suggest our landmark criteria. The resulting landmarks are then used for time warping. Section 3 investigates the variability of the landmark specification before Section 4 provides the analysis for our two datasets. Section 5 concludes the paper. 


\section{Göran Kauermann and Thomas Mestekemper}

\section{Landmark specification}

\subsection{Underlying model}

Before giving our landmark definitions, we introduce our notation and define the underlying model. Let $w_{t}$ be the daily average temperature at time $t$, where time $t$ can be expressed as $t=(i, d)$ with $i$ indexing the year and $d$ giving the day in the year (yearday). We assume that the seasons in year $i$ are compressed and stretched through the monotone function $\pi_{i}(d)$ which describes whether at day $d$ the yearly temperature course is like in the average year, i. e., $\pi_{i}(d)=d$, whether it is running ahead of the average year, i. e., $\pi_{i}(d)<d$, or behind, i. e., $\pi_{i}(d)>d$. Hence, the time transformation $\pi_{i}(d)$ works by accelerating or slowing down time, respectively. The function is called time-warping function subsequently. With $d \in[1,365]$ as day in year we could in principle postulate $\pi_{i}:[1,365] \mapsto[1,365]$, ignoring leap years for simplicity. But in this case, consecutive years would not show a continuous warping structure and we therefore postulate that $\pi_{i}(365)$ and $\pi_{i+1}(1)$ merge continuously. We will come back to this point later. The model is now defined through

$$
w_{t}=\mu_{w}\left(\pi_{i}^{-1}(d)\right)+\epsilon_{t},
$$

where $\mu_{w}(d)$ is the mean temperature at day of the year $d$, averaged over the available years of data. Residual $\epsilon_{t}$ in (2.1) has zero mean but serial correlation is likely and will be taken into account later in the paper. Our intention is now to estimate $\pi_{i}(d)$, preferably in an 'online' manner, i. e., without having full years of data available. To specify function $\pi_{i}(d)$, we allocate a number of landmarks, i. e., time points with clearly defined properties on the data. In a final step, the landmarks will give the information about the warping functions $\pi_{i}(d)$.

\subsection{Temperature thresholds}

A set of easy to calculate landmarks can be located by looking at temperature thresholds, that is we specify the time point when the water temperature course exceeds or falls below a specific value. This can be done with a moving average crossing water temperature threshold. We here pursue an alternative though practically similar idea. We apply simple $t$-tests to the daily mean temperature $w_{\tilde{t}}$ in a small time window $\tilde{t} \in\{t-14, \ldots, t\}$ and test the hypothesis

$$
\mathrm{H}_{0}: \mathrm{E}\left(w_{\tilde{t}}\right) \geq \eta \quad \text { for } \quad \tilde{t} \in\{t-14, \ldots, t\},
$$

against its one-sided alternative. As temperature thresholds we choose $\eta \in\left\{7^{\circ} \mathrm{C}\right.$, $\left.8^{\circ} \mathrm{C}, \ldots, 16^{\circ} \mathrm{C}\right\}$ for the water temperature example and $\eta \in\{4, \ldots, 14\}$ for the air temperature. We set the landmarks for temperature $\eta$ when the corresponding $p$-value of the test crosses the .1 threshold. Exemplarily the $p$-values for the water temperature example at $\eta=7^{\circ} \mathrm{C}, 11^{\circ} \mathrm{C}$ and $15^{\circ} \mathrm{C}$ and the related landmarks are shown in Figure 2. Note that this criterion results in two possible landmarks per 
$p$-value taking into account 15 consecutive days $\left(7^{\circ} \mathrm{C}\right)$

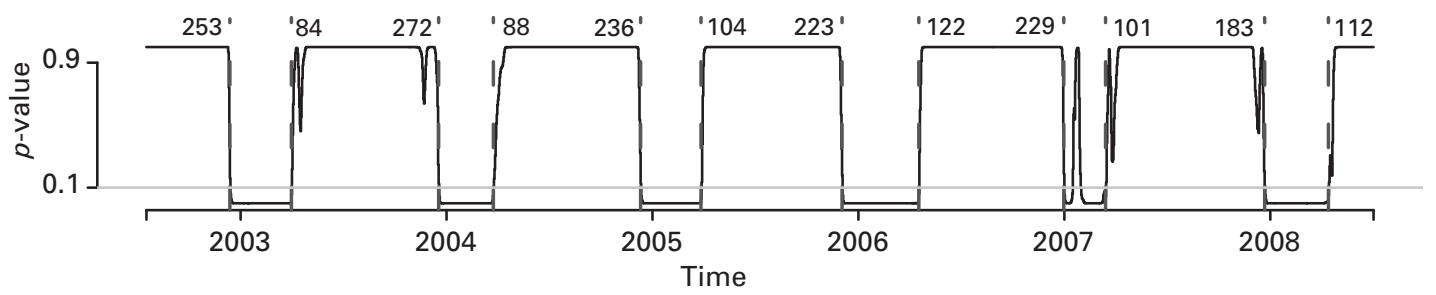

$p$-value taking into account 15 consecutive days $\left(11^{\circ} \mathrm{C}\right)$

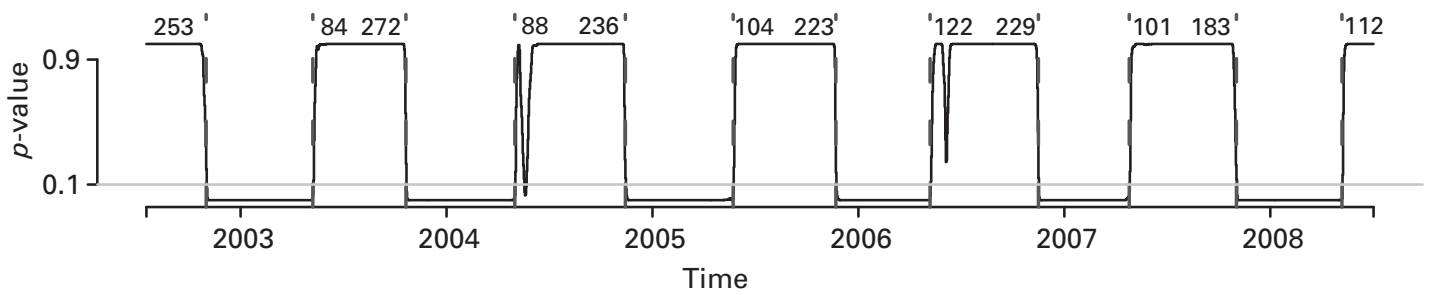

$p$-value taking into account 15 consecutive days $\left(15^{\circ} \mathrm{C}\right)$
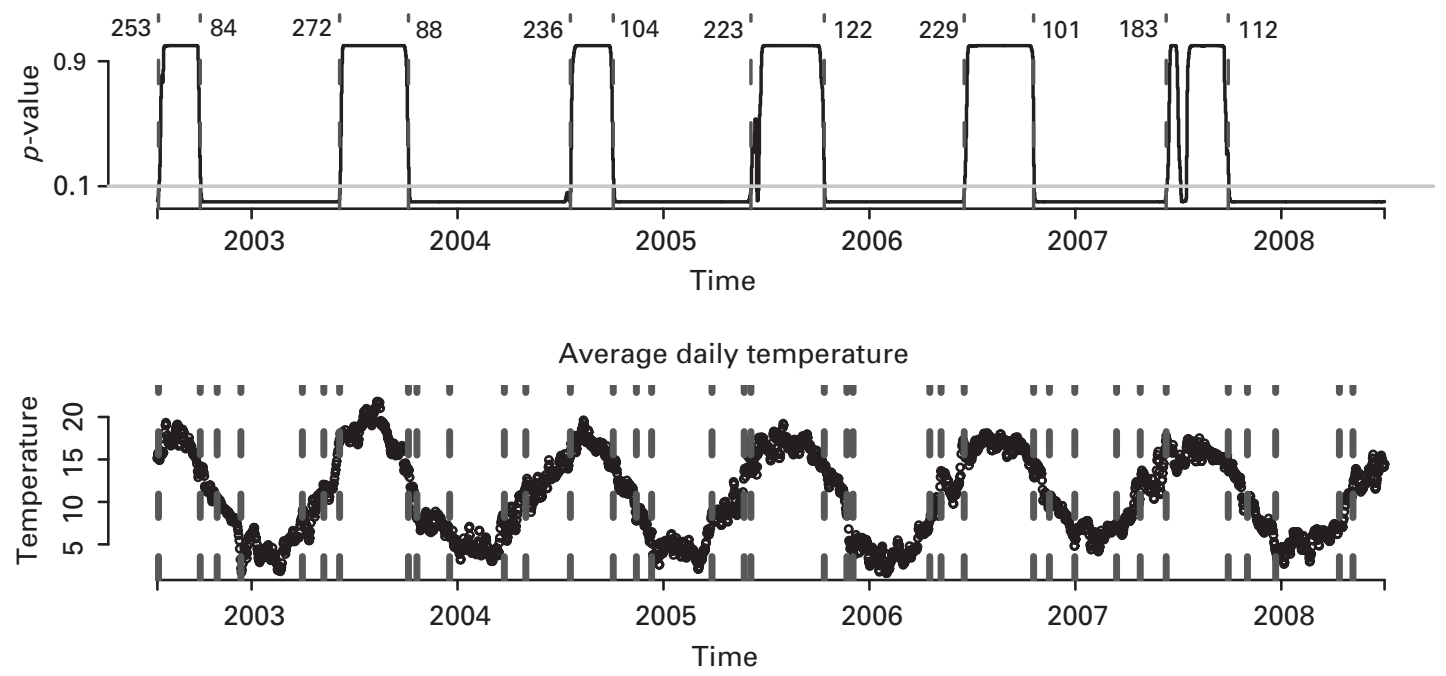

Figure 2 First three panels: $p$-values of the one-sided $t$-tests to hypothesis $(2.2)$ for $\eta=7^{\circ} \mathrm{C}, 11^{\circ} \mathrm{C}, 15^{\circ} \mathrm{C}$ taking into account 15 consecutive days. Bottom panel: daily average water temperature. The vertical dashed lines mark the positions of the daily mean temperature landmarks for $\eta \in\left\{7^{\circ} \mathrm{C}, 11^{\circ} \mathrm{C}, 15^{\circ} \mathrm{C}\right\}$

threshold and per year. The first is set when the $p$-value exceeds the threshold for the first time in the year and the second is set if the $p$-value falls below the threshold for the first time. As we need an online criterion, we only count the first threshold crossing from either side for each year though there might be more than one crossing 
within a small time window. We therefore introduce an additional stability restriction in that we postulate that the minimum distance between two adjacent landmarks for the same value of $\eta$ is 30 days. As a consequence, it can happen that for some years not all landmarks are found. If a landmark is missing, it is simply left out in the calculations below. In total, our approach results in a set of pairs of landmarks based on the average daily temperature which are coherent in terms of monotonicity, i.e., whose sequence is the same for all years.

\subsection{Extreme points}

The temperature threshold approach only provides reliable results during the warming-up phase in spring and the cooling phase in autumn. Consequently, we do not obtain landmarks for the summer or winter months. These are, however, the time windows with the minimum and maximum temperature. We therefore make use of local extrema as further landmark criterion. To pick the exposed extreme points is quite natural for a seasonal time series that follows a roughly sinusoidal pattern. Note, however, that a major drawback is that these landmarks cannot be calculated in a stable way when data arrive sequentially, so that we need sufficient data around the extreme points which is a kind of retrospective point of view. Stable results can be obtained by first smoothing the historical data and then defining landmarks when the derivative of the resulting smooth function crosses zero. We make use of penalized spline smoothing as proposed in Eilers and Marx (1996) and extensively discussed in Ruppert et al. (2003). In fact, the smoothing package SemiPar in R allows to fit a smooth function and its derivative as shown in Figure 3 where the selected landmarks for the water temperature data are given by vertical lines.

Let now $L_{i, k}$ denote the $k$ th landmark in year $i$ where $k$ indexes temperature threshold from Section 2.2 as well as yearly extrema described above. Note that $L_{i, k}$ may exceed the interval $[1,365]$ which happens if a landmark that is normally located near the beginning or end of a year is shifted into the adjacent year. In the next step, we will use the set of landmarks to calculate the time-warping function $\tilde{\pi}(t)$.

\subsection{Landmark-based time warping}

After allocation of the landmarks, we can now focus on our primary goal to estimate the warping functions $\pi_{i}(d)$. We apply a landmark-based curve registration as developed by Kneip and Gasser (1992) and Gasser and Kneip (1995). We therefore rewrite the temperature $w_{t}$ as $w_{i}(d)$ indicating the temperature in year $i$ at day of the year $d$. We assume that the course carries annual characteristics but may be shifted in time. We therefore need a (strictly) monotone time transformation $d \mapsto \pi_{i}(d)$ such that

$$
w_{i}(d)=\mu_{w}\left(\pi_{i}^{-1}(d)\right)+\epsilon_{t},
$$


Average daily temperature

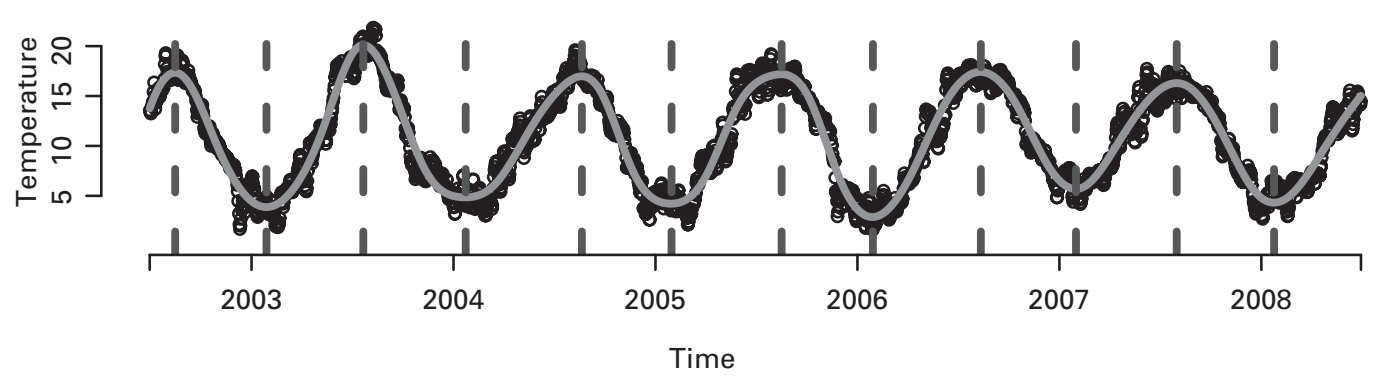

First derivative of the smoothed temperatures

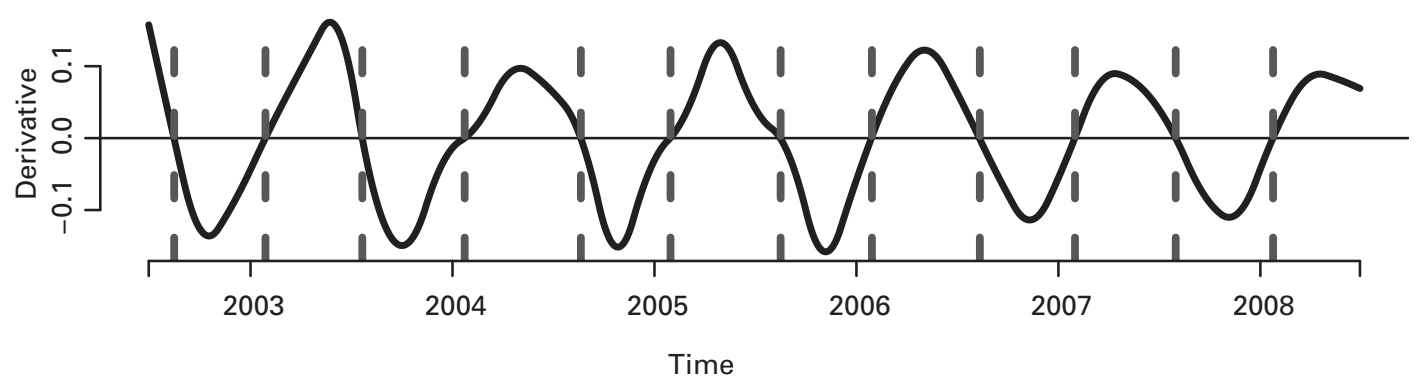

Figure 3 Top panel: the grey line represents the smoothed average daily temperature. Bottom plot: first derivative of the smoothed average daily temperatures. Landmarks are marked by vertical dashed lines

where $\mu_{w}(d)$ is the mean temperature curve that serves as reference for historical and new data. The approach of Kneip and Gasser (1992) has to be extended to our application in so far that the functional observations $w_{i}(d)$ are not independent observations in $i$ but parts from the same time series $w_{t}$. Therefore, we derive a timewarping function $\tilde{\pi}(t)$, say, which holds for the entire data and define $\pi_{i}(d)=\tilde{\pi}(t)$, bearing in mind that time $t$ is indicated by year $i$ and day $d$. That is, instead of estimating time-warping functions for every year of data separately, we fit a single time-warping function for the entire time series. Note that this also allows to apply the routine if data arrive sequentially and we do not need all data and landmarks from an entire year to be able to employ the time-warping procedure. If landmarks in the current year are not yet available, they are simply left out in the calculation of their corresponding reference landmark time point. Reference landmarks are now defined for each $k$ through

$$
\bar{L}_{k}=\frac{1}{I^{\prime}} \sum_{i=1}^{I^{\prime}} L_{i, k},
$$


giving the average location of the historical landmarks over the years. Here $I^{\prime}=I-1$ if the $k$ th landmark has not been reached in the current year, yet, and $I^{\prime}=I$ otherwise with $I$ as current year. The landmarks and their reference points in the entire data series are given by

$$
\bar{L}_{i, k}=(i-1) \cdot 365 \cdot \bar{L}_{k} \quad \text { and } \quad \tilde{L}_{i, k}=(i-1) \cdot 365 \cdot L_{i, k} .
$$

This gives a set of data points $\left(\bar{L}_{i, k}, \tilde{L}_{i, k}\right)$ and time warping assumes that $\tilde{L}_{i, k}=$ $\tilde{\pi}\left(\bar{L}_{i, k}\right)+\epsilon_{i, k}$, where $\tilde{\pi}$ is the monotonic time-warping function. To estimate $\tilde{\pi}(\cdot)$, we apply monotone smoothing to the data points $\left(\bar{L}_{i, k}, \tilde{L}_{i, k}\right)$ following Kelly and Rice (1990). Note that by monotonic smoothing, it is not necessary to impose a coherent ordering restriction on the landmarks, although they were coherent in our data example.

We sketch now how the monotone increasing function $\tilde{\pi}(t)$ can be fitted with penalized splines. To do so we approximate the smoothed time-warping function $\tilde{\pi}(t)$ by a linear combination of a high dimensional B-spline basis of order $q$ through

$$
\tilde{\pi}(t)=\sum_{g=1}^{G} B_{g}(t) u_{g}
$$

where we use quadratic B-splines subsequently built upon equidistant knots. A penalty term on the spline coefficients guarantees a smooth fit, as extensively discussed in Ruppert et al. (2003). Kelly and Rice (1990) point out that there can be no more sign changes in $\tilde{\pi}(t)$ than there are in the sequence of coefficients $\left\{u_{g}\right\}_{g=1, \ldots, G}$. A non-decreasing smooth time-warping function can therefore be found by minimizing the penalized sum of squared residuals

$$
Q(\boldsymbol{u}, \lambda)=\sum_{i=1}^{I} \sum_{k=1}^{K}\left(\tilde{L}_{i, k}-\mathcal{B}\left(\bar{L}_{i, k}\right) \boldsymbol{u}\right)^{2}+\frac{\lambda}{2} \boldsymbol{u}_{t}^{\top} \boldsymbol{R}^{\top} \boldsymbol{R} \boldsymbol{u}^{\top},
$$

subject to the constraints that $u_{1} \leq u_{2} \leq \ldots \leq u_{G}$, where $\mathcal{B}$ denotes the B-spline basis $\left\{B_{g}(t) \mid g=1, \ldots, G\right\}, \boldsymbol{u}=\left(u_{1}, \ldots, u_{G}\right)^{\top}, \lambda$ is a smoothing parameter and $\boldsymbol{R}$ is the second-order difference matrix as suggested in Eilers and Marx (1996), i.e.,

$$
\boldsymbol{R}=\left[\begin{array}{rrrrrr}
1 & -2 & 1 & 0 & 0 & \cdots \\
0 & 1 & -2 & 1 & 0 & \cdots \\
\vdots & \vdots & \ddots & \ddots & \ddots & \ddots
\end{array}\right]
$$

The constraints on the parameter $\boldsymbol{u}$ can be expressed in matrix form $\mathbf{C} \boldsymbol{u} \geq 0$ with obvious definition for $C$. This allows to easily solve the minimization problem (2.4) under the restriction $C \boldsymbol{u} \geq 0$ by quadratic programming. Let therefore $\boldsymbol{u}^{(s)}$ be the current estimate in the $s$ th iteration step and let $\boldsymbol{u}^{(s+1)}=\boldsymbol{u}^{(s)}+\boldsymbol{\delta}^{(s)}$. A Taylor expansion 
of (2.4) yields

$$
Q\left(\boldsymbol{u}^{(s+1)}, \lambda\right)=Q\left(\boldsymbol{u}^{(s)}, \lambda\right)+\underbrace{\frac{\partial Q\left(\boldsymbol{u}^{(s)}, \lambda\right)}{\partial \boldsymbol{u}}}_{\boldsymbol{a}^{(s)}} \boldsymbol{\delta}^{(s)}+\left(\boldsymbol{\delta}^{(s)}\right) \underbrace{\frac{\partial^{2} Q\left(\boldsymbol{u}^{(s)}, \lambda\right)}{\partial(\boldsymbol{u})^{\top} \partial \boldsymbol{u}}}_{\boldsymbol{B}^{(s)}} \boldsymbol{\delta}^{(s)},
$$

so that we have to minimize the quadratic form

$$
\left(a^{(s)}\right)^{\top} \boldsymbol{\delta}^{(s)}+\left(\boldsymbol{\delta}^{(s)}\right)^{\top} \boldsymbol{B} \boldsymbol{\delta}^{(s)},
$$

subject to $\boldsymbol{C} \boldsymbol{\delta}^{(s)} \geq-\boldsymbol{C} \boldsymbol{u}^{(s)}$. An alternative approach to monotone smoothing with quadratic B-splines using linear programming for $L_{1}$-optimization can be found in He and Shi (1998).

It remains to choose the smoothing parameter $\lambda$. We suggest to minimize the Akaike Information Criterion given by

$$
\operatorname{AIC}(\lambda)=Q(u, 0)+2 \cdot \operatorname{df}(\lambda),
$$

where the number of degrees of freedom is defined to be the trace of the smoother matrix

$$
\operatorname{df}(\lambda)=\operatorname{tr}\left(\mathcal{B}\left(\mathcal{B}^{\top} \mathcal{B}+\lambda R^{\top} R\right)^{-1} \mathcal{B}^{\top}\right)
$$

For our data examples, we employed a B-spline basis with 10 knots per year. For the water temperature, the smoothing parameter was chosen to be $\lambda=0.01579$ and the resulting fit is shown as solid lines in Figure 4. If a season is running ahead of its correspondent in the reference year, we find $\pi_{i}(d)$ below the diagonal. A course above the diagonal means that the season is running late. For example, the winter in 2006 came late and did not last long as it merged with an early spring in 2007.

\section{Variability of the time-warping function}

The calculated landmarks itself are random variables and their specification is therefore stochastic. This induces stochastic variability for the estimation of the warping function which needs to be assessed on statistical grounds. It is difficult to derive the variability of the estimation of $\pi_{i}(d)$, analytically, so that we instead look at the variability of the landmarks first. In order to assess the variability of the landmark specifications, we run a bootstrap procedure. The idea is to use model (2.1) in order to bootstrap the time series $w_{t}$. From the newly generated series, we can recalculate the landmarks and derive a bootstrapped time-warping function. This allows us to assess the variability of the time-warping function. 
Year $2002 / 2003$

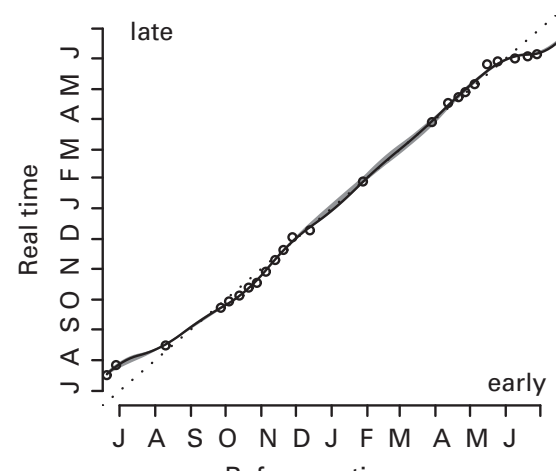

Reference time

Year 2004/2005

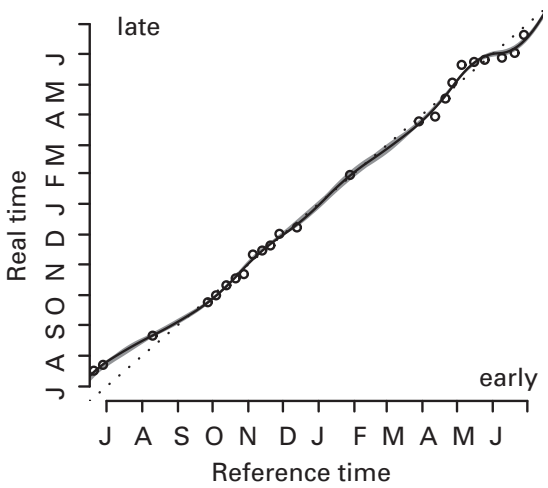

Year 2006/2007

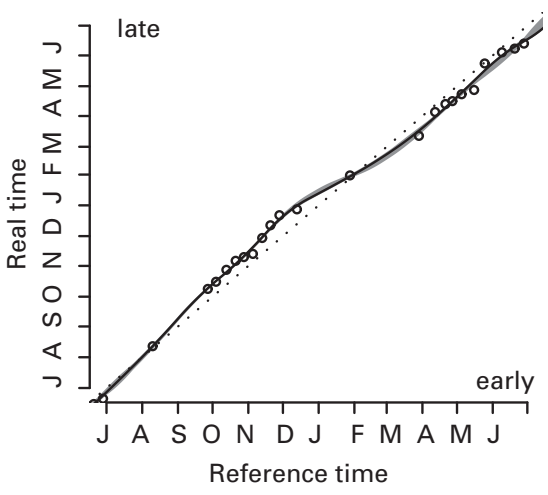

Year 2003/2004

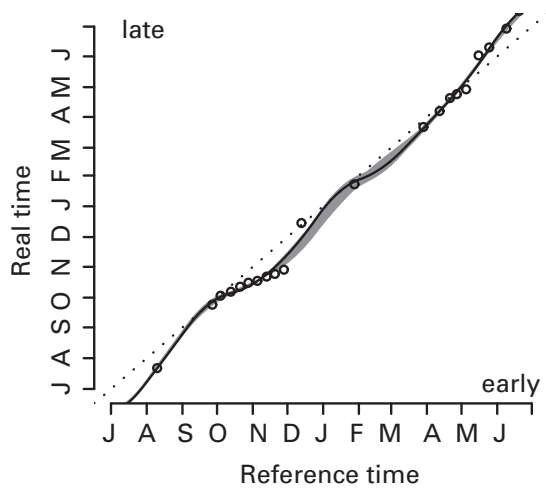

Year 2005/2006

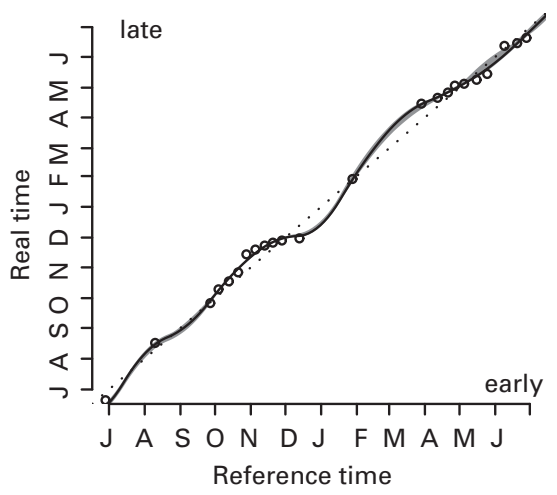

Year 2007/2008

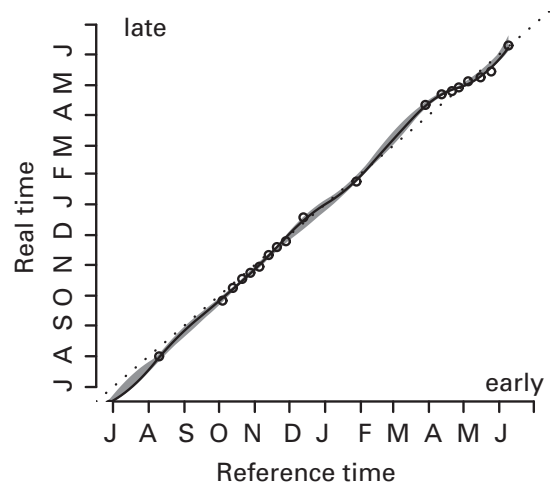

Figure 4 Course of the time-warping functions $\hat{\pi}_{i}(d)$ for the six years of our dataset (black solid lines). The grey band marks the area where all bootstrapped time-warping functions $\hat{\pi}_{i}^{*}(d)$ where contained in. Observed landmarks are shown visualized by circles 
For the bootstrap, we use model (2.3) but allow the residual process $\epsilon_{t}$ to carry serial correlation. We therefore assume an autoregressive model

$$
\epsilon_{t}=\sum_{j=1}^{J} \alpha_{j} \epsilon_{t-j}+e_{t},
$$

where $\alpha_{k}$ are autocorrelation coefficients and $e_{t}$ is a white noise residual process upon which we build our bootstrap. Note that $e_{t}$ may still carry some heterogeneity and we therefore pursue a wild bootstrap approach (Mammen, 1993). That is, we draw the bootstrapped residual $e_{t}^{*}$ from a two-point distribution given by $\hat{e}_{t} \cdot((1-$ $\sqrt{5}) / 2,(1+\sqrt{5}) / 2)$ with probabilities $((5+\sqrt{5}) / 10,(5-\sqrt{5}) / 10)$, where $\hat{e}_{t}$ are the fitted residuals based on (3.1). Inserting the resulting bootstrap in the autoregressive model gives

$$
\epsilon_{t}^{*}=\sum_{j=1}^{J} \alpha_{j} \epsilon_{t-j}^{*}+e_{t}^{*},
$$

relying on the $J$ initial values $\epsilon_{1}, \epsilon_{2}, \ldots, \epsilon_{J}$. We then obtain the bootstrapped time series by setting

$$
w_{t}^{*}=\mu_{w}\left(\pi_{i}^{-1}(d)\right)+\epsilon_{t}^{*} .
$$

Applying the landmark criteria from Section 2.2 to this newly generated time-series yields a set of bootstrapped landmarks and thereby allows the estimation of a bootstrapped time-warping function $\tilde{\pi}^{*}(t)$.

\section{Data examples}

\subsection{Water temperature}

We first investigate the water temperature data shown in Figure 1. The warping functions are already shown in Figure 4. To apply the bootstrap, we first have to calculate the mean temperature function $\mu_{w}(d)$. We therefore use the warped mean daily temperature functions $w\left(\hat{\pi}_{i}(d)\right)$ shown in Figure 5. Calculating the crosssectional mean of these curves would lead to a wiggly estimation of $\mu_{w}(d)$. To avoid this, we instead smooth over the warped temperature courses again using a B-spline basis but now in cyclic form with six equidistant knots over the year. The resulting estimation $\hat{\mu}_{w}(d)$ is illustrated in Figure 5 as superimposed smooth curve.

We obtain residuals $\hat{\epsilon}_{t}$ by subtracting the re-warped mean function $\hat{\mu}_{w}\left(\hat{\pi}_{i}^{-1}(d)\right)$ from the observed daily mean temperature curves. Next, we use the autoregressive model (3.1), where the autoregressive order was set to $J=3$ based on the partial autocorrelation function. We took the empirical residuals $\hat{e}_{t}$ and employed the wild bootstrap routine as described above to generate 500 new time series. From 
Warped temperature curves and mean temperature function

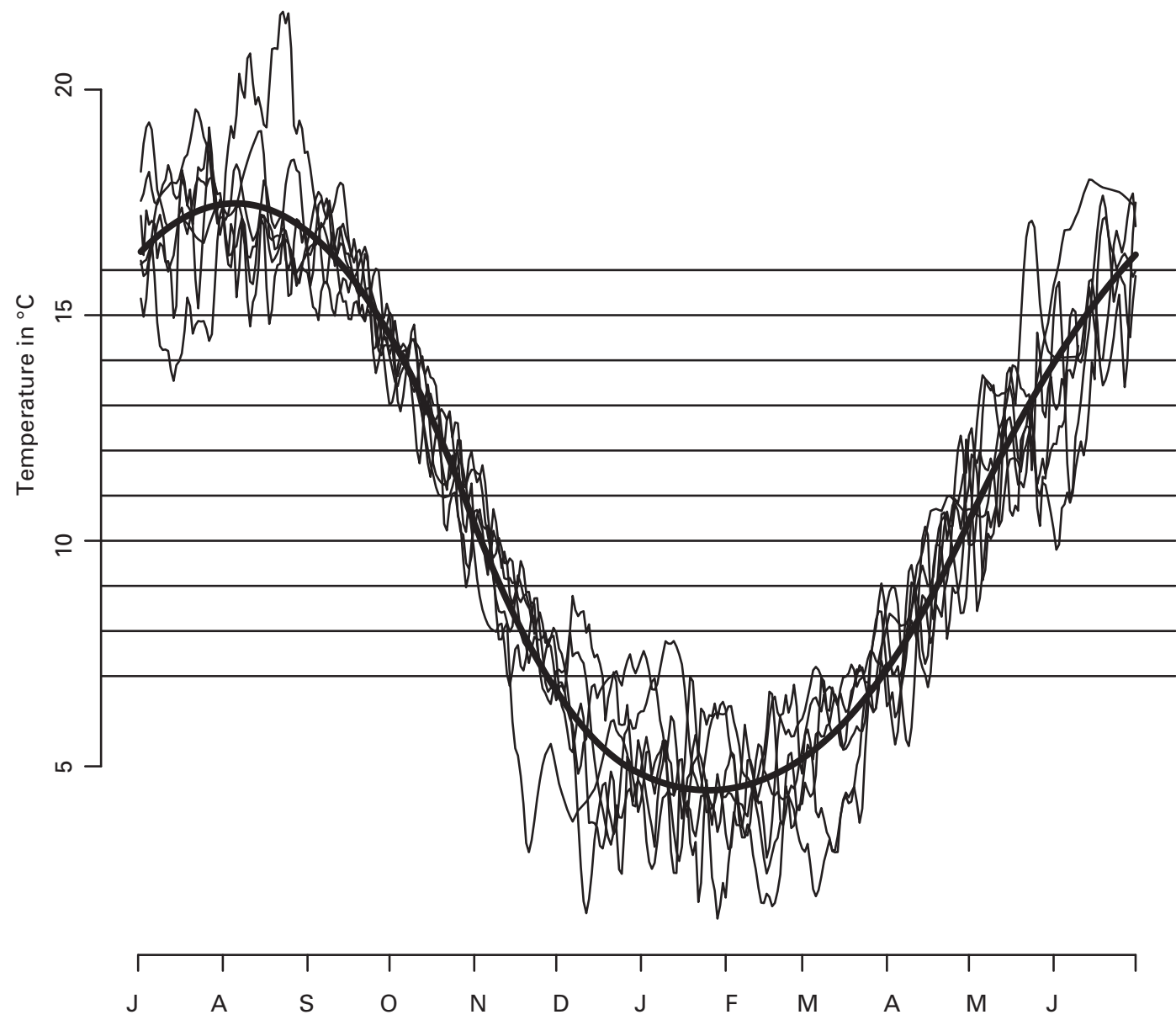

Day of the year

Figure 5 Warped daily mean temperature courses $\bar{w}\left(\hat{\pi}_{i}(d)\right), i=1, \ldots, 6$ with smoothed mean temperature function $\mu_{w}(d)$ superimposed. Vertical lines indicate the temperature thresholds that were used for the landmark calculation

each we calculated the bootstrapped time-warping functions $\pi_{i}^{*}(d)$ by employing the monotonic smoothing described above. The grey shaded space in Figure 4 gives the area where all 500 bootstrap replica were contained in. The variability is small except for 2003/2004. Overall, we can now derive statements about the yearly shifts of seasonal pattern as tentatively formulated in Section 1. That is summer 2003 started early and lasted long (May to September) or winter 2005 started early. 


\subsection{Air temperature}

As second example we explore air temperature data collected at the (former) airport Berlin-Tempelhof in Germany. The data at hand give the average air temperature from 01.01.1991 to 08.12.2010. In the analysis of this dataset, we focus on the 'online' use of the warping idea. We therefore use the data from year 2010 to show how to make use of the above calculation sequentially in time. Starting from 2010, we recalculate the warping function each time a landmark in 2010 has been reached. Figure 6 shows these warping functions, where we plot the deviation to the reference time as separate fitted lines which successively extend the warping function to the right. The points thereby indicate the landmarks. In the graph, we superimpose the bootstrap interval calculated from the entire dataset. We see that the variability of the bootstrap mirrors the variability of the sequential warping functions.

To be more specific, we investigate the variability of the warping function estimates if new data, that is landmarks, become available. Let therefore $L_{1}<L_{2}<\ldots$ define the successively occurring landmarks in 2010 and define with $\hat{\pi}_{L_{k}}(t)$ the warping function estimate calculated at landmark time point $L_{k}$ with $t \leq L_{k}$. We may look at $\hat{\pi}_{L_{k}}\left(L_{j}\right)$ for $j \leq k$ and assess how the estimates vary with increasing $k$. That is we look at the landmark time points (real time) in Figure 6 and investigate how the fitted warping functions vary with further landmarks becoming available. Estimates $\hat{\pi}_{L_{k}}\left(L_{j}\right)$ for $j \leq k$ are plotted against $L_{k}$ in Figure 7 , upper plot. The resulting empirical standard deviation of $\hat{\pi}_{L_{k}}\left(L_{j}\right)$ for $j<k$ is shown in the bottom plot of Figure 7. Overall, the estimates $\hat{\pi}_{L_{k}}\left(L_{j}\right)$ vary with about two to three days of

Year 2010

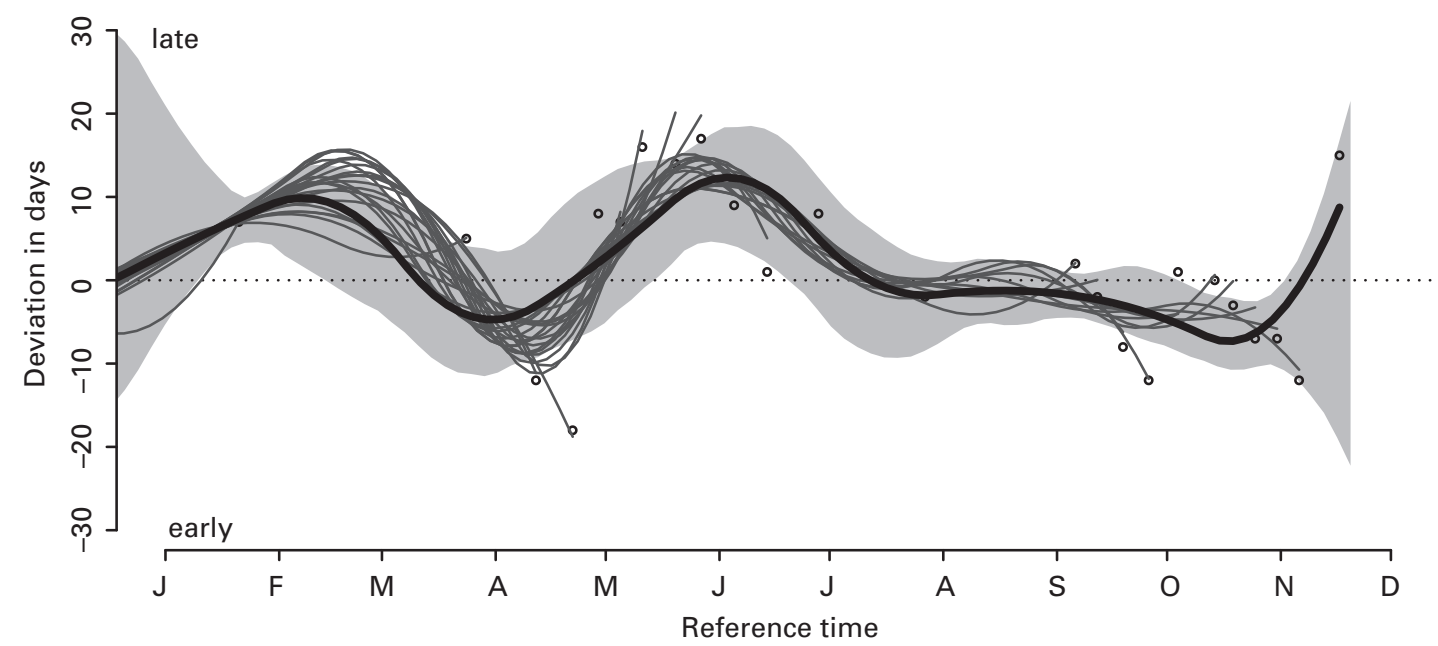

Figure 6 Deviation of fitted warping functions from average year, calculated sequentially with each new occurring landmark. A bootstrap interval is superimposed 


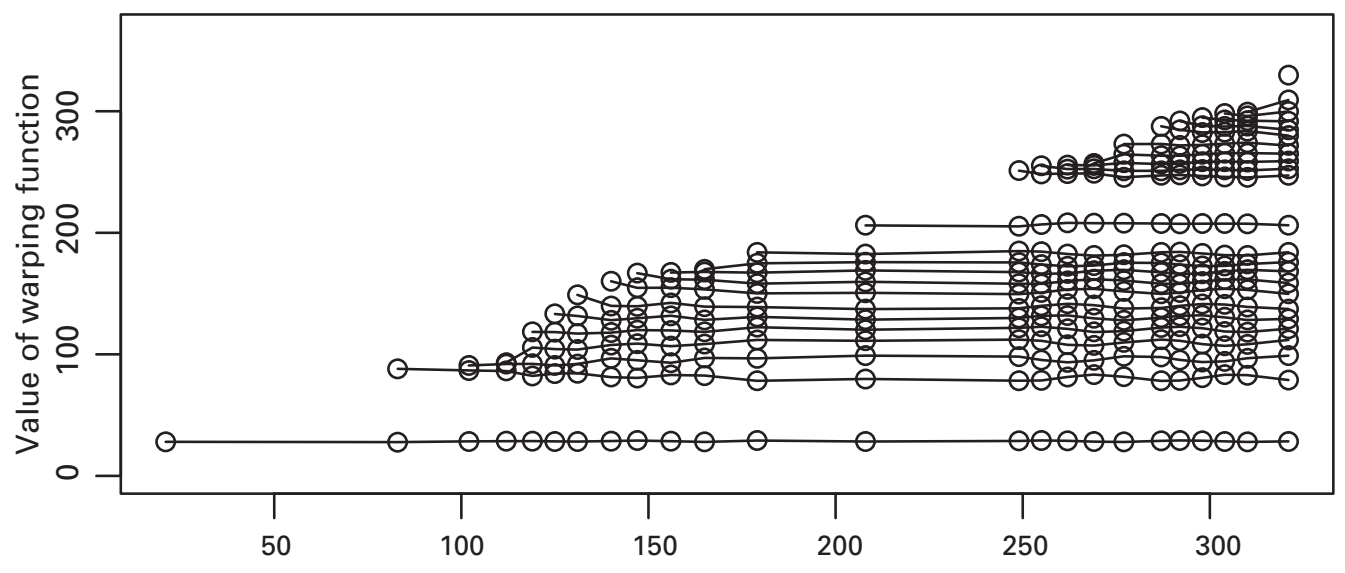

Day in 2010

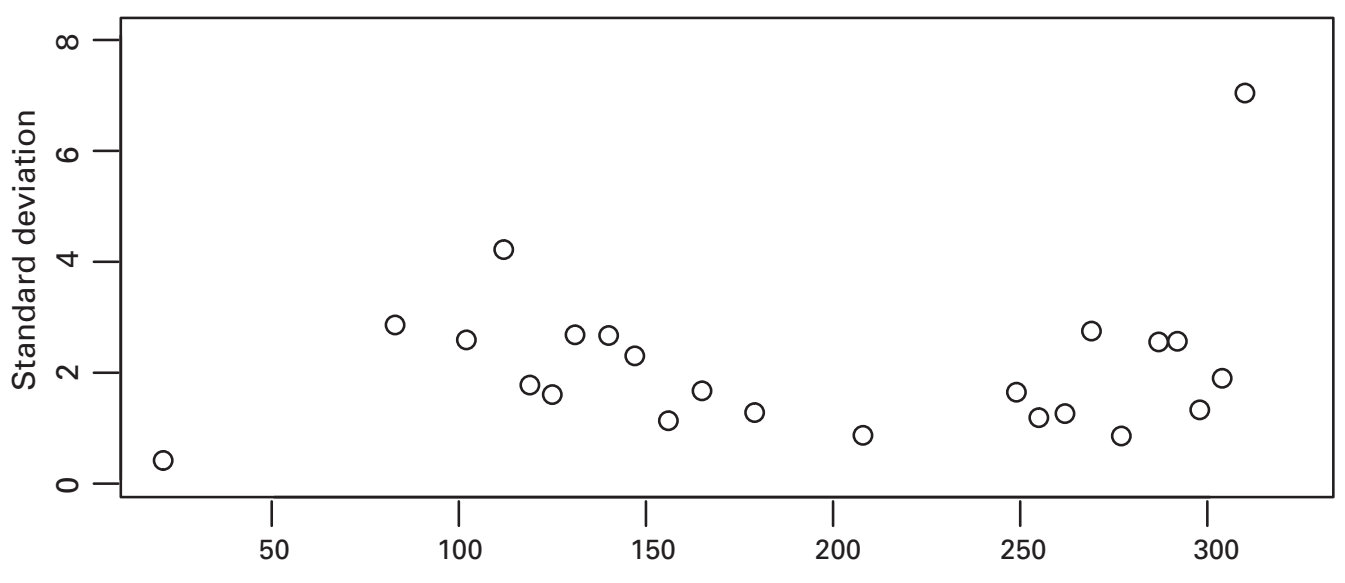

Day in 2010

Figure 7 Estimates $\hat{\pi}_{L_{k}}\left(L_{j}\right)$ for $j \leq k$ (upper plot) and their resulting empirical standard deviation

standard deviations, so that the warping function calculated in a sequentially or 'online' manner is rather stable.

\section{Discussion}

In this paper, we demonstrate landmark specification and time warping for temperature data recorded in subsequent years. The proposed method allows to assess whether the season in a year is running ahead or behind the average. The method 
allows to be applied online in the sense that not the entire data of a complete year are necessary to draw the warping function. A bootstrap-based procedure is suggested to assess the estimation variability. Overall, the proposed method is simple but effective, so that it may allow to be generally used for consecutive temperature recording.

\section{References}

Bigot J (2006) Landmark-based registration of curves via the continous wavelet transform. Journal of Computational and Graphical Statistics, 15, 542-64.

Eilers PHC and Marx BD (1996) Flexible smoothing with B-splines and penalties. Statistical Science 11, 89-121.

Ferraty F and Vieu P (2006) Nonparametric functional data analysis: theory and practice. New York: Springer.

Gasser T and Kneip A (1995) Searching for structure in curve samples. Journal of the american statistical association, 90, 1179-88.

Gervini G and Gasser T (2004) Self-modelling warping functions. Journal of the Royal Statistical Society - Series B, 66, 959-71.

He X and Shi P (1998) Monotone B-spline smoothing. Journal of the American Statistical Association, 93, 643-50.

Kelly C and Rice J (1990) Monotone smoothing with application to dose-response curves and the assessment of synergism. Biometrics, 46, 1071-85.

Kneip A and Gasser T (1992) Statistical tools to analyze data representing a sample of curves. The Annals of Statistics, 20, 1266-305.

Liu X and Yang MCK (2009) Simultaneous curve registration and clustering for functional data. Computational Statistics and Data Analysis, 53, 1361-76.

Mammen E (1993) Bootstrap and wild bootstrap for high dimensional linear models. Annals of Statistics, 21, 255-85.

Ramsay JO, Hooker G and Graves S (2009) Functional data analysis with $R$ and $M A T L A B$. New York: Springer.

Ramsay JO and Li X (1998) Curve registration. Journal of the Royal Statistical Society Series B, 60, 351-63.

Ramsay JO and Silverman BW (2005) Functional data analysis. New York: Springer.

Rønn BB (2001) Nonparametric maximum likelihood estimation for shifted curves. Journal of the Royal Statistical Society Series B, 63, 243-59.

Ruppert D, Wand M and Carroll R (2003) Semiparametric regression. Cambridge, UK: Cambridge University Press.

Silverman BW (1995) Incorporating parametric effects into principal components analysis. Journal of the Royal Statistical Society Series B, 57, 673-89. 
